\title{
Integrative model of the response of yeast to osmotic shock
}

\author{
Edda Klipp ${ }^{1,5}$, Bodil Nordlander ${ }^{2,5}$, Roland Krüger ${ }^{3}$, Peter Gennemark ${ }^{4}$ \& Stefan Hohmann ${ }^{2}$
}

Integration of experimental studies with mathematical modeling allows insight into systems properties, prediction of perturbation effects and generation of hypotheses for further research. We present a comprehensive mathematical description of the cellular response of yeast to hyperosmotic shock. The model integrates a biochemical reaction network comprising receptor stimulation, mitogen-activated protein kinase cascade dynamics, activation of gene expression and adaptation of cellular metabolism with a thermodynamic description of volume regulation and osmotic pressure. Simulations agree well with experimental results obtained under different stress conditions or with specific mutants. The model is predictive since it suggests previously unrecognized features of the system with respect to osmolyte accumulation and feedback control, as confirmed with experiments. The mathematical description presented is a valuable tool for future studies on osmoregulation in yeast and-with appropriate modifications-other organisms. It also serves as a starting point for a comprehensive description 을 of cellular signaling.

Osmoregulation encompasses active processes with which cells monitor and adjust osmotic pressure and control shape, turgor and relative water content. Even individual cells in multicellular organisms respond to osmotic changes, and strategies of cellular adaptation are conserved from bacteria to human ${ }^{1}$. The yeast Saccharomyces cerevisiae is a suitable model system to study osmoregulation and a substantial amount of information is available on osmotic shockinduced signal transduction, control of gene expression and accumulation of osmolytes ${ }^{2}$.

Osmoregulation is a homeostatic process, though commonly studied as a response to osmotic shock. Central to yeast osmotic adaptation is the high osmolarity glycerol (HOG) signaling system ${ }^{2,3}$ (Fig. 1). $S$. cerevisiae monitors osmotic changes through the plasma membrane-localized sensor histidine kinase Sln1. Under ambient conditions, $\operatorname{Sln} 1$ is active and inhibits signaling. Upon loss of turgor pressure, $\operatorname{Sln} 1$ is inactivated ${ }^{4}$ resulting in activation of a mitogen-activated protein (MAP) kinase cascade and phosphorylation of the MAP kinase Hog1. Active Hog1 accumulates in the nucleus where it affects gene expression. Two HOG target genes encode enzymes in glycerol production. Hence, activation of Hogl stimulates the production of glycerol, which serves as an osmolyte to increase intracellular osmotic pressure. Glycerol accumulation is also controlled by rapid closing of the aquaglyceroporin Fps1, which is an osmolarity-regulated glycerol channel. Hog1 activation and Hog1-dependent transcriptional stimulation are transient processes, indicating rigorous feedback control. Several protein phosphatases are known as negative regulators of the pathway. Although the overall organization of the systems is well characterized, open questions concern the mechanisms underlying activation and deactivation of the system, feedback control and the causal relationship between different events in osmotic adaptation.

To investigate the dynamics and logic of the response of yeast cells to osmotic shock, we developed a mathematical model. Previously, quantitative computational simulation proved to support understanding of regulatory systems ${ }^{5-10}$, characterizing threshold properties and bistability ${ }^{11,12}$, characteristic times ${ }^{13,14}$ and feedback effects ${ }^{12,15-19}$. The model presented here comprises receptor stimulation, the HOG signaling pathway, activation of gene expression, adaptation of cellular metabolism, glycerol accumulation and a thermodynamic description of the control of volume and osmotic pressure. This integration of the biochemical reaction network with the biophysical description of cellular volume control is crucial for understanding the activation and downregulation of the signaling pathway. Using a combination of experimental studies and simulations, we describe mechanisms underlying feedback control of the HOG pathway in osmotic adaptation. We provide evidence that turgor recovery due to glycerol accumulation mediates pathway downregulation and that this mechanism ensures the important combination of effective feedback control with competence for restimulation by subsequent osmotic treatments.

Certain events in osmotic adaptation, such as the relative levels of activated Hog1, target gene mRNA as well as enzyme and glycerol levels have been quantified experimentally in time courses. For many steps in the signaling cascade, such data cannot currently be generated. The model assists in predicting the behavior of those steps, allows identification of system features, permits simulation of different experimental conditions and thereby directs hypotheses framing for further experimental research.

\footnotetext{
${ }^{1}$ Berlin Center for Genome Based Bioinformatics (BCB), Max-Planck Institute for Molecular Genetics, Dept. Vertebrate Genomics, Ihnestr. 73 , 14195 Berlin, Germany. ${ }^{2}$ Department of Cell and Molecular Biology/Microbiology, Göteborg University, Box 462, S-40530 Göteborg, Sweden. ${ }^{3}$ Humboldt University Berlin, Institute for Biology, Invalidenstr. 43, 10115 Berlin, Germany. ${ }^{4}$ Department of Computer Science and Engineering, Chalmers University of Technology, S-41296 Göteborg, Sweden. ${ }^{5}$ These authors contributed equally to this work. Correspondence should be addressed to E.K. (klipp@molgen.mpg.de) or S.H. (hohmann@gmm.gu.se).
}

Published online 17 July 2005; doi:10.1038/nbt1114 


\section{RESULTS}

Existing quantitative and time course data for many of the biomolecules involved in the osmotic shock response are too sparse to allow mathematically satisfactory parameter determination. To overcome this limitation and to structure available information, we divided the system into functional modules as a critical level of biological organization (Fig. 1) ${ }^{20}$ : (i) the phosphorelay module including the sensor Sln1, (ii) the MAP kinase cascade, (iii) the gene expression module, (iv) the metabolism module including glycerol efflux and (v) biophysical changes. The Sho1-Ste11 branch of the HOG pathway, which operates in parallel with the $\operatorname{Sin} 1$ branch to activate Pbs2, was not considered because the $\operatorname{Sln} 1$ branch in isolation exhibits a similar response profile as the entire system under the conditions considered ${ }^{21}$. The model for the entire reaction network consists of 32 ordinary differential equations (ODE) and 70 parameters plus three additional ODE and two algebraic equations for changes in volume, osmotic pressure and turgor pressure $\Pi_{t}$ and for water flow over the cell membrane (Supplementary Tables 1-5 online).

To develop and test the model in a systematic fashion, we first analyzed the behavior of individual modules in steady state. Subsequently, a standard time course experiment was performed and the data were used to estimate parameters such that simulations matched the behavior observed in experiments (Supplementary Fig. 1 online). 윽 Finally, to test the model and to gain understanding of regulatory principles of the osmotic stress response, we analyzed effects of physiological and genetic perturbations both by simulation and experiments.

\section{Steady states of individual modules}

Phosphorelay module. Its switch-like, input-output characteristics are crucial for the entire response. They can be assessed from the dependence of the steady-state concentration of Ssk1 on the kinetic parameter of $\operatorname{Sin} 1$ autophosphorylation, $k_{1}^{T C S}$, which represents the degree of turgor pressure loss (Fig. 2a and equation (1)). The yeast phosphorelay module consists of three proteins (Sln1, Ypd1 and Ssk1) as compared to two proteins in bacterial systems. We compared steady-state characteristics for phosphorelay systems consisting of one, two or three proteins (Fig. 2a). A lower number of components led to smoother dependence of the output signal on $k_{1}^{T C S}$, whereas the switch-like behavior became more pronounced for higher numbers of components.

MAP kinase module. The steady-state concentration of phosphorylated $\operatorname{Hog} 1\left(\operatorname{Hog} 1 \mathrm{P}_{2}\right)$ is a function of the input signal $($ Ssk1) and the ratio $k_{+} / k_{-}$, where $k_{+}$and $k_{-}$are the rate constants of all kinases and phosphatases, respectively. This ratio determines the level of $\mathrm{Hog} 1 \mathrm{P}_{2}$ in the presence or absence of stress. Upon stress, low $k_{+} / k_{-}$ratios cause high absolute $\operatorname{Hog} 1 \mathrm{P}_{2}$ values with low signal amplification, whereas high ratios result in low $\mathrm{Hog} 1 \mathrm{P}_{2}$ with the potential for strong amplification (Supplementary Fig. 2 online).

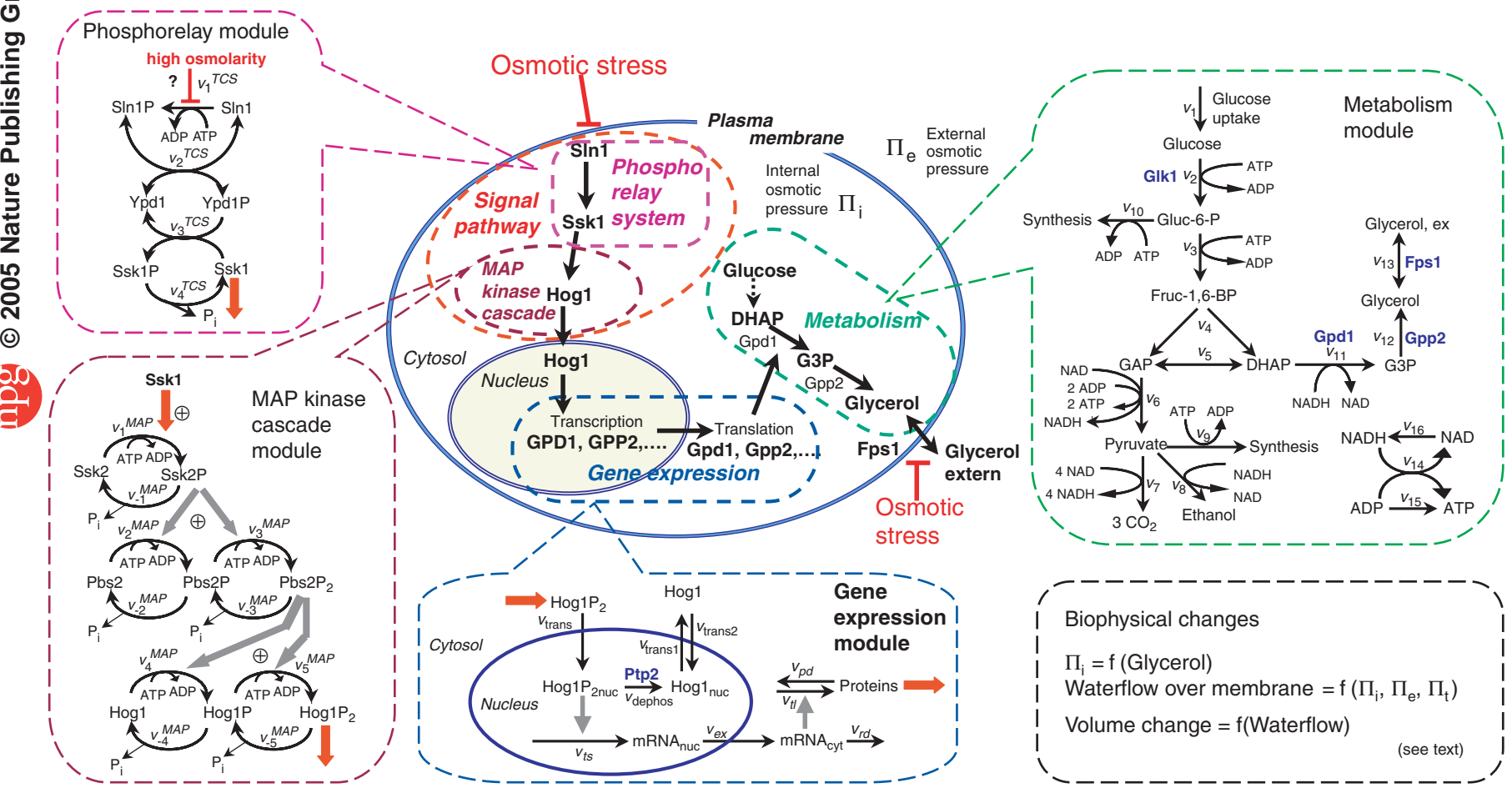

Figure 1 Overview of the response of yeast to hyperosmotic stress. The membrane-localized histidine kinase $\operatorname{SIn} 1$ senses osmotic stress. Upon increase in external osmotic pressure SIn1 is inactivated leading to accumulation of dephosphorylated Ssk1, which is an activator of the MAPKKK Ssk2 (and the redundant Ssk22). Ssk2 activates by phosphorylation the MAP kinase Pbs2, which in turn phosphorylates and activates the MAP kinase Hog1. $\mathrm{Hog} 1 \mathrm{P}_{2}$ enters the nucleus to activate gene expression. Hog 1 is inactivated by dephosphorylation mediated by nuclear as well as cytosolic protein phosphatases. Two of the genes whose expression is stimulated by active Hog1 code for the enzymes Gpd1 and Gpp2, which catalyze the conversion of dihydroxyacetonephosphate (DHAP) via glycerol-3-phosphate (G3P) to glycerol. Closure of the osmotically sensitive glycerol channel Fps1 supports glycerol accumulation, which increases internal osmotic pressure. Internal and external osmotic pressures are mainly determined by solute concentrations. Their changes cause altered turgor pressure, which is mediated by the elasticity of plasma membrane and cell wall and by water flow over the membrane. Both SIn 1 and Fps 1 sense turgor pressure changes. For mathematical modeling, the system was divided into the following modules: the phosphorelay module, the MAP kinase cascade, the transcription and translation module, the module for volume and pressure changes, and the metabolism module. The identifiers $v \ldots$ denote the reaction rates, which were calculated using equations detailed in Supplementary Tables 1-4. 
Metabolism module. The intracellular glycerol concentration $\mathrm{Glyc}_{\text {in }}$ is determined by the rates of glycerol production and efflux. The steadystate concentration of glycerol at varying enzyme concentrations or varying flux through Fps1 ( $\left.\mathrm{v}_{13}\right)$ were compared (Fig. 2b). A closure of the channel alone led to an increase in $\mathrm{Glyc}_{\text {in }}$ owing to basal level production, whereas an increase in enzyme concentrations alone had almost no effect on $\mathrm{Glyc}_{\mathrm{in}}$, because increased production was balanced by outflow; this is consistent with experimental observations of Fps1 mutants unable to close. The combination of closing the Fps1 channel plus upregulation of glycerol production caused a marked increase in $\mathrm{Glyc}_{\text {in }}$ at steady states.

\section{Time course experiments}

The standard experiment is a single osmotic shock with $0.5 \mathrm{M} \mathrm{NaCl}$ where $\operatorname{Hog} 1 \mathrm{P}_{2}$, GPD1 (or STL1) mRNA, Gpd1 protein (enzyme activity) as well as intracellular and total glycerol is monitored over time (Fig. 3). This was simulated starting with a low constant osmotic pressure for the time $t<0$ and higher osmotic pressure following addition of $0.5 \mathrm{M} \mathrm{NaCl}$ for $t \geq 0$ (Fig. 3a,c,e,f). Figure 3b,d shows experimental data.

In the simulation, osmotic shock caused an immediate increase of active Ssk1 (no experimental time course data is available) owing to water outflow and a drop in $\Pi_{t}$ (Fig. 3f). The subsequent decline of Ssk1 coincided with the commencement of glycerol accumulation and 을 hence the time point when turgor started to increase again (Fig. 3a,f).

The signal is further transmitted by phosphorylation of Ssk2 and Pbs2. Hog $1 P_{2}$ increased more slowly than Ssk1. The concentrations of GPD1 mRNA and Gpd1 increased and declined with a certain time delay (Fig. 3a). Glyc in assumed an intermediary level after about 30 min. At this time total glycerol levels started to rise owing to reopening of the Fps1 channel. Interestingly, the initial increase in Glyc in was mainly due to closure of Fps1 at basal glycerol production (Fig. 3c). Enhanced glycerol production caused by the increase in Gpdl level mainly contributed to maintaining high Glyc $\mathrm{c}_{\text {in }}$.

The simulation of biophysical quantities is shown in Figure 3e,f. Upon osmotic shock (increase of $\Pi_{e}$ ) $\Pi_{t}$ dropped immediately and adapted during $45 \mathrm{~min}$. When the final value for $\Pi_{t}$ was reached, Fps 1 progressively reopened to release glycerol, which prevented further turgor increase. The cell volume dropped markedly upon osmotic shock owing to water outflow, in accordance with experimental data (for Hansenula anomala ${ }^{22}$ ). During adaptation the volume increased because of water influx without reaching its initial value, which is in line with experimental observations ${ }^{23} . \Pi_{t}$ and volume remained below initial values since (i) $\Pi_{t}$ already passed the threshold for pathway activation (compare Fig. 2a), and (ii) Fpsl opened gradually to release glycerol thereby controlling further increase of $\Pi_{t}$.

Deactivation of the HOG pathway is achieved by two different mechanisms. (i) Elevated Glycin caused an increase of $\Pi_{i}$ and thus water influx, cell swelling and increased turgor pressure. This effect activated Sln1, causing Ssk1 inactivation, which in turn resulted in decline of pathway activation. (ii) Phosphatases of the signaling pathway constantly dephosphorylate $\mathrm{Hog} 1 \mathrm{P}_{2}$ thereby counteracting pathway stimulation of the pathway. Once pathway activation was turned off, phosphatases caused inactivation of Hog1 and hence pathway downregulation. Although the model contains a moderate upregulation of phosphatase activity in accordance with experimental reports ${ }^{24}$, a very similar pathway downregulation was observed when phosphatase activity was kept constant in simulations (data not shown). We performed a sensitivity analysis to test the influence of the parameter choice on the quality of the fit (Supplementary Fig. 3 online) and on the timing and strength of response (Supplementary Fig. 4 online).

\section{Situations not considered in model development}

Model performance was tested using data from system perturbations available in the literature or from experiments conducted in this study. For this, the numerically estimated parameters for the standard experiment were used.

Different degrees of osmotic shock. Treatments with progressively increasing $\mathrm{NaCl}$ concentrations (from $0.1 \mathrm{M}$ to $1.5 \mathrm{M}$ ) were predicted in simulations to result in progressively lower initial cell volume, consistent with published data ${ }^{23}$. For moderate pathway stimulation, peak levels of $\operatorname{Hog} 1 \mathrm{P}_{2}$ and mRNA of HOG-dependent genes were lower compared to the standard experiment. Since only the $\operatorname{Sin} 1$ branch is included in the model, these experiments were performed using a $\Delta$ ste 11 mutant, which lacks Shol branch activity. Experimental data (Supplementary Table 6 online) and simulation showed gradually increasing amplitudes of $\mathrm{Hog} 1 \mathrm{P}_{2}$ with increasing $\mathrm{NaCl}$ concentrations, approaching a maximal level. The maximal amplitude was, however, reached at lower concentrations in the experiment than in simulations.
Figure 2 Steady states of the phosphorelay module and the metabolism module. (a) Phosphorelay module: output signal (concentration of Ssk1) plotted against input signal $k_{1}^{T C S}$ (curve a). In the connected model the change of $k_{1}^{T C S}$ is related to the change of turgor pressure $\Pi_{t}$ (equation (1)). Upon variation of $k_{1}^{\text {TCS }}$ the value for Ssk 1 changes only slightly as long as $k_{1}^{T C S}$ is above a certain threshold, while exhibiting a strong increase once $k_{1}^{T C S}$ is below this threshold. Then the signaling pathway is 'on,' although its activity could still be modulated by further decrease of $k_{1}^{\text {TCS }}$. Hence, the system shows a pronounced switch-like behavior. The SIn1-Ypd1-Ssk1 module consists of three proteins as compared to two proteins in bacterial phosphorelay systems. The input-output behavior for a two-protein system is depicted in curve $b$ and for a single protein system in curve $c$. A larger number of components results in a sharper switch. (b) Metabolism module: dependence of the glycerol concentration on the kinetic constant of the Fps1 aquaglyceroporin (numbers: value of $k_{13}$ in $s^{-1}$ ) and on the level of Gpd1 (given as relative values compared to the pre-stress steady state; the Gpd1 levels correlate to glycerol production capacity). In the pre-stress steady state, $k_{13}$ has the value $5.10^{-3} \mathrm{~s}^{-1}$. Upon stress Fps 1 closes and hence $k_{13}$ is assumed to decrease. Accumulation of glycerol depends both on the glycerol production capacity and on the ability to close Fps1 and retain glycerol. The marginal increase in the external glycerol level has not been taken into account here.
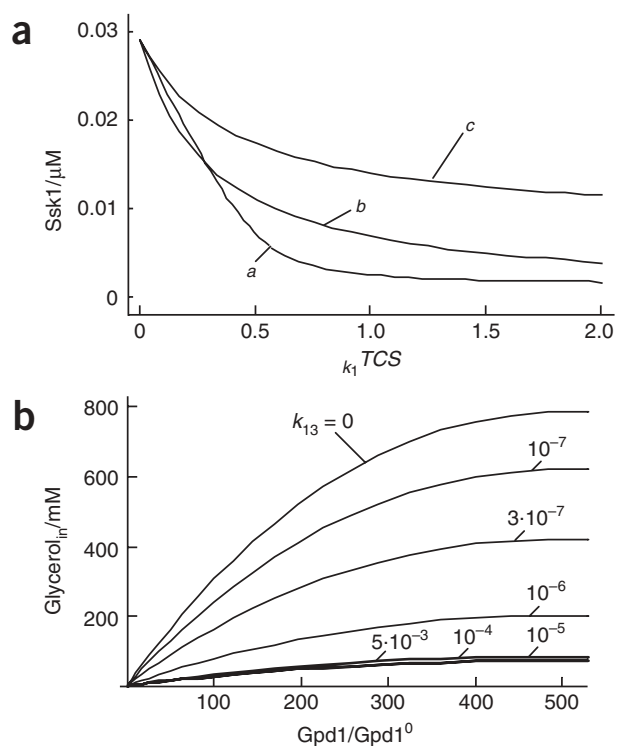
Figure 3 Time courses for key molecules of the osmotic shock response monitored in the standard experiment, that is, a single osmotic shock with $0.5 \mathrm{M} \mathrm{NaCl}$ at time 0 min.

(a) Relative concentrations of molecules involved in signaling and metabolic adaptation: Ssk1 (gray dashed line), total $\mathrm{Hog}_{1} \mathrm{P}_{2}$ (black solid line), generic HOG-dependent mRNA (gray solid line), and protein-product of a gene whose expression is stimulated (black dashed-dotted line). Relative ने concentrations are normalized with respect to the maximal value attained during the time course of the standard experiment. (b) Experimentally determined time courses (relative values) of total $\mathrm{Hog}_{1} \mathrm{P}_{2}$ (black solid line, circles), mRNA of a generic HOG-dependent gene (gray solid line, triangles), and the specific activity of the enzyme Gpd1 (black dashed-dotted line, squares).

(c) Simulated glycerol concentrations in the cell (Glycin; black line) and in the medium (Glyc $\mathrm{C}_{\mathrm{ex}}$; gray dashed line). (d) Experimentally determined glycerol concentrations inside cells (black line,

filled circles) and in the medium (gray line, open circles). (e) Simulated cellular volume $V$ (relative values). (f) Simulated turgor pressure $\Pi_{t}$ (gray dashed line), internal osmotic pressure $\Pi_{i}$ (black

solid line) and external osmotic pressure $\Pi_{e}$ (dark dashed line) during osmotic shock and

윽 adaptation. Upon osmotic stress, turgor pressure

drops immediately. This leads to water outflow and a rapid volume decrease. Signaling

molecules respond sequentially in response to turgor pressure loss: first the output signal of the phosphorelay system, Ssk1, is activated, and then the MAP kinase Hog1 is phosphorylated and subsequently the concentrations of mRNA, protein, and eventually glycerol rise. When turgor pressure starts normalizing, the signaling pathway is switched off in the same sequence as observed for activation. Panels a,c,e,f: Simulations with parameters optimized on the basis of the experimental data. Panels $\mathbf{b}$ and $\mathbf{d}$ : Experimental data (see also Supplementary Fig. 1 only).

For stronger osmotic stress, the response amplitude was reached at a later time point and the response period increased (Supplementary in Fig. 5 online), consistent with published data ${ }^{25,26}$.

() Mutants unable to produce or accumulate glycerol. The $\Delta g p d 1$ $\Delta g p d 2$ double mutant is unable to produce glycerol ${ }^{27}$. The mRNA in Figure 4a may be of any Hog1-dependent gene. Activation of the system caused a strongly prolonged increase in $\mathrm{Hog} 1 \mathrm{P}_{2}$ and mRNA with less pronounced downregulation, in agreement with experimental data (Fig. 4b). Since $\mathrm{Hog} 1 \mathrm{P}_{2}$ activates phosphatase transcription approximately twofold, a slight pathway downregulation occurs. In a different scenario an Fps1 unable to close upon hyperosmotic shock was simulated (Fig. 4c). The increase in $\mathrm{Hog}_{1} \mathrm{P}_{2}$ and mRNA was again prolonged until glycerol accumulation eventually occurred, resulting in increased turgor and pathway deactivation after a long delay. Also this is in agreement with experimental data (Fig. 4d and Supplementary Fig. 6 online) although we consistently observed that the $\mathrm{Hog}_{1} \mathrm{P}_{2}$ level fluctuated. As cells expressing unregulated Fps1 overproduced glycerol, it was the inability to accumulate glycerol, rather than to produce it, which caused prolonged HOG pathway activity.

Glycerol overproduction. To simulate the inverse situation, that is, faster glycerol accumulation, we increased the GPD1 mRNA production rate. This resulted in similar amplitudes of $\mathrm{Hog}_{1} \mathrm{P}_{2}$ and HOGdependent mRNA but the period of activation was shorter, consistent with experimental data ${ }^{28}$ (Supplementary Fig. 7 online).

Enhanced phosphatase activity. Simulating the osmotic shock profile with a fourfold higher protein phosphatase level resulted in a similar period of HOG pathway activity but diminished amplitudes of $\mathrm{Hog}_{1} \mathrm{P}_{2}$ and mRNA. Experimentally this was tested by overexpressing PTP2 from the GAL1 promoter ${ }^{29}$. The experimentally observed profile was similar to that obtained in simulations (Supplementary Fig. 8 online). To further test the effect of phosphatase overproduction, we combined fourfold increased phosphatase levels with unregulated Fps1. Although enhanced phosphatase activity reduced the amplitude of HOG pathway activity, it could not prevent the prolonged stimulation of $\mathrm{Hog}_{1} \mathrm{P}_{2}$ and mRNA. Taken together, enhanced expression of Ptp2 altered the amplitude, but not the period of HOG pathway activity after osmotic shock. This conclusion is different from that reached in a recent report suggesting that phosphatases are more important for signal duration than kinases ${ }^{30}$.

Pathway reactivation. A critical test of the model was simulation of two subsequent osmotic shocks addressing the questions whether a second shock can reactivate the pathway. This was simulated by treating cells twice at different time intervals $(15,30$ and $60 \mathrm{~min})$ with $0.5 \mathrm{M} \mathrm{NaCl}$ (Fig. 5 and Supplementary Fig. 9 online). For a 15-min interval the system behaved as it does after a single stimulation with $1 \mathrm{M} \mathrm{NaCl}$. However, for a longer interval between the two treatments, two separate peaks of similar profile were observed. Also in experiments, the HOG pathway could readily be reactivated by a second osmotic treatment and hence does not become desensitized under continuous osmostress. The feedback control mechanisms operate such that they downregulate the system while allowing for full responsiveness. When we performed simulations where HOG pathway activation mediated more than twofold stimulation of the 
Figure 4 Test of model predictions and effects of system perturbations. Using the parameters estimated for the standard experiment (see Figure $\mathbf{3}$ and Supplementary Tables $\mathbf{1 - 5}$ online) the effects on the system caused by different osmotic stress conditions and specific mutations were studied in simulations (left panels) and in experiments (right panels). (a) Time course simulations for selected compounds in a $\triangle g p d 1$ $\triangle g p d 2$ double mutant, which is unable to

خे produce glycerol. Compounds include total $\mathrm{Hog} 1 \mathrm{P}_{2}$ (black solid line), mRNA (gray solid line), protein concentration (black dashed-dotted line) of a generic HOG-dependent gene and glycerol concentration (gray dotted line). The mutation is implemented in the model by setting $v_{11}=0$. (b) Experimental time courses for the $\triangle g p d 1$ 1 $\Delta g p d 2$ double mutant: relative values of total $\mathrm{Hog} 1 \mathrm{P}_{2}$ (black solid line, circles) and STL1 mRNA (gray solid line, triangles). Because $4 g p d 1$ $\Delta g p d 2$ mutant cells cannot produce glycerol they are unable to adapt to the loss of turgor pressure proteins), but not switched off, although a moderate decrease following the initial peak is observed. This partial downregulation is due to a moderate HOGdependent stimulation of phosphatase levels. (c) Time course simulations for yeast cells unable to close the Fps1 glycerol channel following osmotic shock: $\mathrm{Hog} 1 \mathrm{P}_{2}$ (black solid line), mRNA (gray solid line), protein concentration (black dashed-dotted line), and glycerol concentration (gray dotted line). The mutation is implemented in the model by keeping $k_{13}=0.1 \mathrm{~s}^{-1}$ unaltered during simulations. (d) Experimental time courses for yeast cells unable to close the Fps 1 glycerol channel following osmotic shock: relative changes for total Hog $1 \mathrm{P}_{2}$ (black solid line, circles), STL1 mRNA (gray solid line, triangles) and Gpd1 specific activity (black dashed-dotted line, squares). In contrast to the $4 g p d 1$ Agpd2 double mutant, which cannot produce glycerol, the strain (Supplementary Fig. 6 online). However, like the $4 g p d 1$ ugpd2 double mutant, cells with constantly open Fps1 are unable to rapidly accumulate glycerol. The consequence is similar in both cases: the pathway remains activated for a longer period.

protein phosphatase activity, such pathway reactivation was not observed (data not shown).

The model also correctly simulated experiments where the HOG pathway was activated by genetic manipulation (Supplementary Fig. 10 online). a

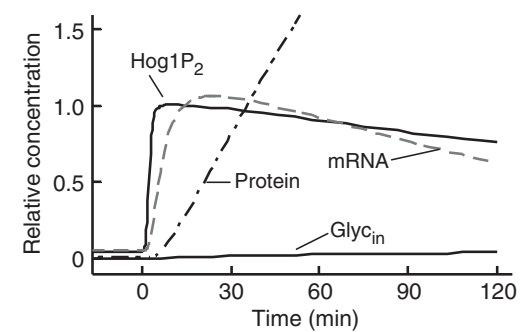

b

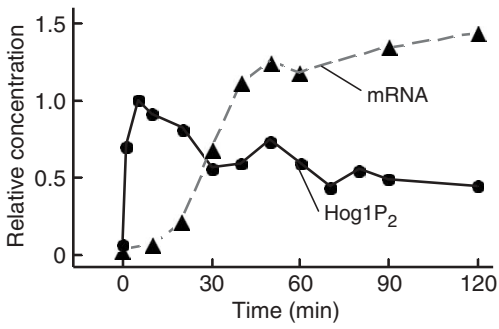

d

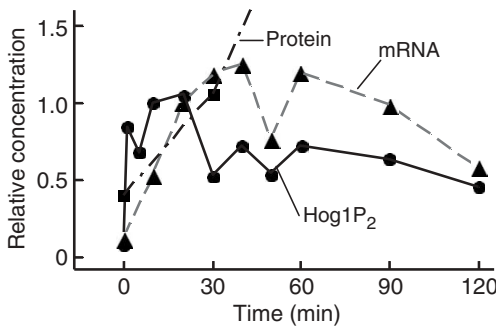

Figure 5 Test of model predictions for the experimental scenario of two subsequent osmotic shock treatments at various time points. In all panels: first stimulation with $0.5 \mathrm{M} \mathrm{NaCl}$ at $t=0 \mathrm{~min}$, followed by a second stimulation with an additional $0.5 \mathrm{M} \mathrm{NaCl}$ at $15 \mathrm{~min}, 30 \mathrm{~min}$ or $60 \mathrm{~min}$. Single, only one stimulation (standard experiment). The parameters are those estimated for the standard experiment (single osmotic shock with $0.5 \mathrm{M} \mathrm{NaCl}$; Supplementary Tables 1-5 online). (a,b) Relative concentrations of total $H_{0 g} \mathrm{P}_{2}$. (c,d) Relative mRNA concentrations for a generic HOG-dependent gene (GPD1 in experimental data). (e,f) Intracellular glycerol concentrations. Simulations $(\mathbf{a}, \mathbf{c}, \mathbf{e})$ are compared with experimental time courses $(\mathbf{b}, \mathbf{d}, \mathbf{f})$. Simulations and experiments show that the pathway remains competent for a second osmotic stimulation at different stages of adaptation to a first osmotic treatment. If the second shock is applied very soon (15 min) after the first one, the profiles of Hog1 phosphorylation and Hog1-dependent mRNA levels resemble that of a single shock with twice the amount of $\mathrm{NaCl}$. The second shock also leads to additional glycerol accumulation.
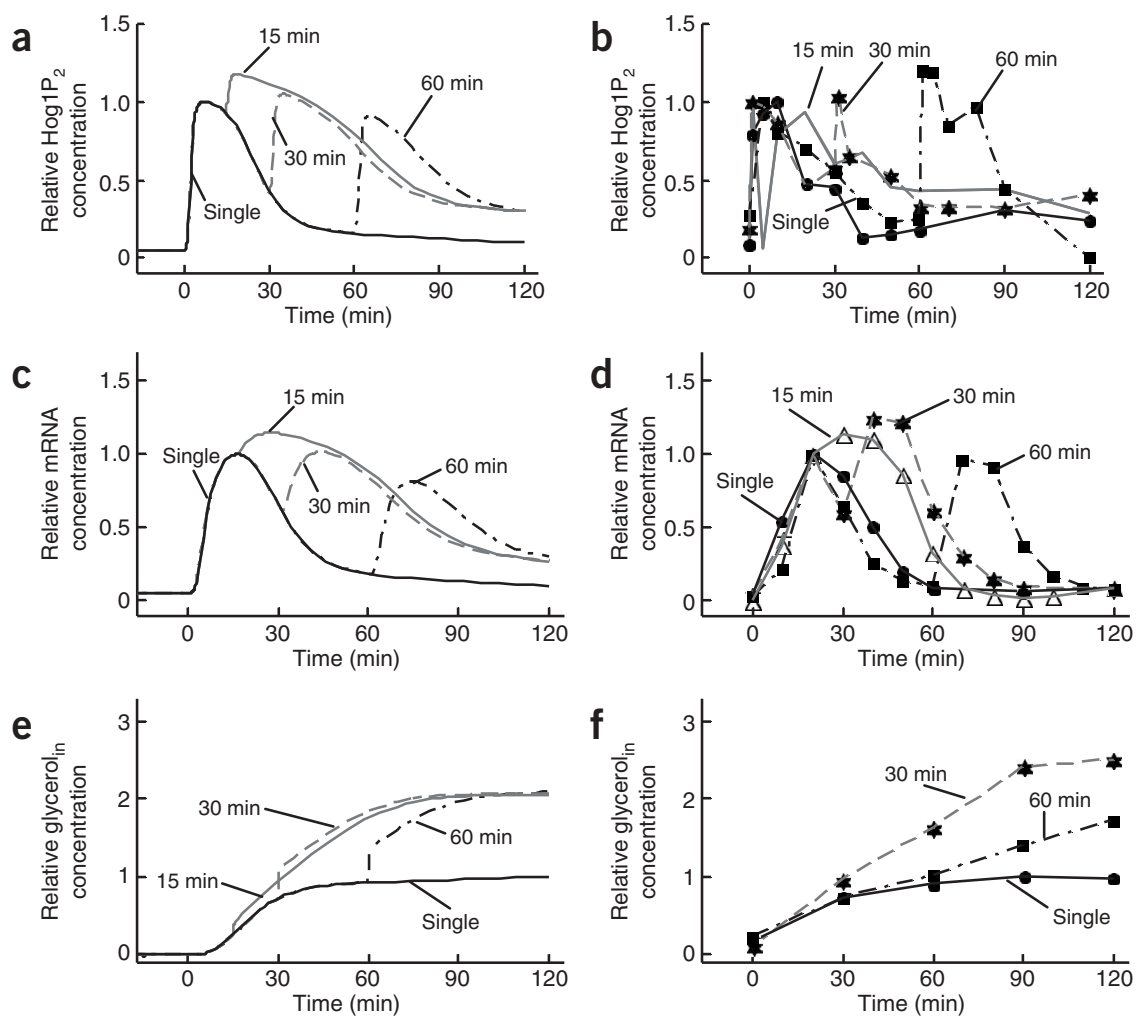
osmoregulation in other organisms. Future studies will also help to iteratively improve the presented model.

Our study had three aims. First, we wished to generate a mathematical description of yeast osmoregulation that reproduced experimental observations reasonably well. Simulations took into account steady states and time courses after single and repeated osmotic treatments, and the effects of genetic perturbations. Simulations agreed well with experimental data, where we monitored pathway output and physiological effects. Therefore the biological information, the model assumptions, and the parameters used to match experimental data appear to be of sufficient precision for a reliable mathematical description.

The second aim was to extract novel information on the dynamic operation of the processes underlying osmotic adaptation. Three examples of particular interest are illustrated here.

The design of the eukaryotic phosphorelay module Sln1-Ypd1-Ssk1 is different from bacterial two-component systems containing two instead of one phosphotransfer step. One reason may be to pass the signal from cell surface to nucleus with the help of the phosphotransfer protein $\mathrm{Ypd}^{11,32}$. On the other hand, an additional phosphotransfer step allows the system to attain a sharper, switch-like behavior such that it remains unresponsive until a certain threshold. Above this threshold, the amplitude increases progressively approaching a maximum. At higher stress intensity the period of stimulation is 윽 progressively prolonged.

Another feature emerging from our simulations concerns the crucial role of the Fps1 aquaglyceroporin ${ }^{33}$ in controlling glycerol accumulation and therefore also controlling signaling through the HOG pathway. The basal glycerol production level combined with rapid closure of Fps1 is sufficient to explain an initial glycerol accumulation after osmotic shock, which in turn accounts for HOG pathway downregulation. This is in agreement with the experiments showing that inability to accumulate glycerol, such as in cells expressing unregulated Fps1, results in strongly prolonged HOG pathway activation. It is generally assumed that stimulated expression of GPD1 and GPP2 and the resulting increased glycerol production capacity accounts for the increase in intracellular glycerol levels. Simulation suggests that this effect is instead important for maintaining a high level of glycerol production in adapted cells. Further studies can now address the relative roles of transport and gene expression on osmolyte accumulation. Interestingly, aquaglyceroporins of the characteristics of Fps1 have been found only in yeasts so far $^{34}$. Given the importance of such osmolyte transporters in modulating the osmotic response and turgor pressure, it seems likely that proteins of similar role should exist in other organisms as well.

Another important conclusion from simulations and experiments concerns feedback regulation of the HOG pathway. It has been suggested that enhanced expression of genes encoding phosphatases accounts for feedback control ${ }^{2}$. Using different variants of the model, we observed normal feedback even without invoking an increased phosphatase activity, which hence does not seem necessary to downregulate the pathway. Importantly, the pathway can be fully reactivated by a second osmotic shock. According to simulations this would not be possible if phosphatase levels increased more than about twofold and if this were the main mechanism for feedback control. This is in accordance with experimental data, which indicate that the transcript levels of phosphatase genes increases by no more than twofold. Moreover, it appears that a transcriptional activation of phosphatases is too slow to explain the observed feedback pattern ${ }^{35}$. Simulations and experimental data argue that downregulation is mediated by glycerol accumulation, cell reswelling and increase in turgor. Interestingly, simulations and parallel monitoring of different events in osmotic adaptation in wild type and mutants with delayed glycerol accumulation (Supplementary Fig. 6 online) show that pathway downregulation occurs already at about one-third of the maximal glycerol level, that is, before cells are fully adapted. Probably, the commencement of cell reswelling provides the signal that reverses activation of the osmosensors. This concept is supported by recent experimental data $^{36}$ obtained using certain mutants in an experimental system of "conditional osmotic stress," showing that cells adapt at different rates depending on the rate of uptake of external osmolytes. Taken together, this suggests that the main role of the phosphatases is to constantly counteract pathway activation to set thresholds and reduce noise instead of providing a pathway-intrinsic feedback loop. However, slightly enhanced phosphatase expression may account for the observed slow pathway downregulation following osmotic shock in mutants unable to produce glycerol.

Our third aim was to generate a model that can be further optimized iteratively together with experimental studies, thereby serving as a tool for hypothesis-driven experimental studies. Predictions based on the simulations presented here have already been tested in experiments. If the model is to be further developed and a more accurate mathematical description of osmoregulation obtained, it is crucial to generate quantitative data for steady states and transient processes. Ongoing modeling attempts aim to include kinetic details of individual processes as well as cellular compartmentation and spatial effects of signaling. Striving for a comprehensive understanding of systems properties of osmoregulation and signaling in general constitutes a significant challenge in quantitative experimental biology as well as computational systems biology.

\section{METHODS}

Below we present an explanation of the mathematical modeling. Experimental materials and methods are described in the Supplementary Data online.

Modeling strategy. The dynamics of a biochemical reaction system is described by a set of ordinary differential equations (ODEs)

$$
d c_{i} / d t=\sum_{j=1}^{r} n_{i j} v_{j}(i=1, \ldots, m),
$$

where $m$ is the number of biochemical species with the concentrations $c_{i}$ and $r$ is the number of reactions with the rates $v_{j}$, and the quantities $n_{i j}$ denote the stoichiometric coefficients. The equations are given in Supplementary Tables 1-5. All numerical calculations have been carried out using Mathematica Version 4.1, Wolfram Research.

The complete system and its modules are shown in Figure 1. All modules were described with kinetic models using linear, bilinear or Michaelis-Mententype kinetics, resulting in a nonlinear system of ODEs. Their stoichiometry, steady states and dynamic behavior were analyzed separately. Initial parameters were determined to match the experimentally observed behavior of the modules. Kinetic parameters for the metabolism module as well as some parameters for the biophysical description were taken from literature ${ }^{37,38}$. The remaining parameters were manually selected from analysis of the individual modules (such as steady-state characteristics described above and characteristic times of pathway activation or deactivation). Subsequently, modules were connected for a comprehensive view of the process. Data obtained for the standard osmotic shock experiment (wild-type cells treated with $0.5 \mathrm{M} \mathrm{NaCl}$; Supplementary Fig. 1 and Supplementary Table 6 online) were used to fine tune the parameters. The ODE system was simulated to obtain time curves for all components included in the model. The general qualitative behavior of the model was robust to small and even intermediate changes of parameters, providing confidence that plausible parameter values were obtained (see Supplementary Data for a more detailed discussion of parameter dependence). Spatial effects were not considered at this stage and we assumed that signaling 
compounds diffuse sufficiently quickly to acquire a uniform distribution. We further assumed that the system is in steady state before osmotic shock.

Individual modules. The phosphorelay system (for equations and parameters see Supplementary Table 1 online). We considered four reactions: autophosphorylation of $\operatorname{Sln} 1\left(v_{1}^{T C S}\right)$, reversible phosphotransfer to $\operatorname{Ypd} 1\left(v_{2}^{T C S}\right)$, phosphotransfer to Ssk1 $\left(v_{3}^{T C S}\right)$, and dephosphorylation of Ssk1 $\left(v_{4}^{T C S}\right)$. The alteration of turgor pressure, $\Pi_{t}(t)$, is the input signal for this module and the concentration of unphosphorylated Ssk1 is the output signal. The block of Sln1 autophosphorylation upon osmotic shock was implemented via a dependence of the kinetic parameter $k_{1}^{T C S}$ (which enters $v_{1}^{T C S}$ linearly) on turgor pressure:

$$
k_{1}^{T C S}(t)=k_{1}^{T C S, 0} \cdot\left(\frac{\Pi_{t}(t)}{\Pi_{t}^{0}}\right)^{n_{1}}
$$

The precise dependence of $\operatorname{Sn} 1$ autophosphorylation on turgor pressure is not known from experiments. Equation (1) was chosen since it ensured that (i) activation of the phosphorelay module only depends on the value of the turgor pressure at any given time, (ii) $k_{1}^{T C S}$ increases steadily with $\Pi_{t}$. Termination of the signaling process requires an increase of $\Pi_{t}(t)$ as a consequence of cellular adaptation. For the connected model, where changes in turgor pressure eventually depend on the glycerol concentration, equation (1) implements a negative feedback loop from the glycerol level to the activation of the phosphorelay module.

The MAP kinase cascade (Supplementary Table 2 online). The active form of Ssk1 constitutes the link to the MAP kinase cascade. The module comprises the following reactions: Ssk1-dependent autophosphorylation of Ssk2 (representing the pool of redundant proteins Ssk2 and Ssk22) $\left(v_{1}^{M A P}\right)$; phosphorylated Ssk2 phosphorylates Pbs2 on two sites $\left(v_{2}^{M A P}\right.$ and $\left.v_{3}^{M A P}\right)$; double-phosphorylated Pbs2 phosphorylates Hog1 on two sites $\left(v_{4}^{M A P}\right.$ and $\left.v_{5}^{M A P}\right)$ and thereby activates it. All phosphorylated compounds are dephosphorylated by protein phosphatases $\left(v_{-i}^{M A P}, i=1, \ldots, 5\right) . \operatorname{Hog} 1 \mathrm{P}_{2}$ can be dephosphorylated in the cytosol or, alternatively, can enter the nucleus $\left(v_{\text {trans }}\right)$, but is not considered to leave it. $\mathrm{Hog} 1 \mathrm{P}_{2}$ can be dephosphorylated in the nucleus $\left(v_{\text {dephos }}\right)$ and unphosphorylated Hog1 leaves the nucleus $\left(v_{\text {trans } 1,2}\right)$. Hog $1 \mathrm{P}_{2 \text { nuc }}$ is the output signal of this module and we considered it as a transcription factor.

Transcription and translation (Supplementary Table 3 online). To simplify the complexity of transcription and translation, we assumed equal concentrations and rates of change for all HOG-dependent mRNAs and proteins except

20 of those for the activated phosphatases. The rate of mRNA formation $\left(v_{\mathrm{ts}}\right)$ is dependent on the basal activity of the corresponding gene and modulated by the concentration of $\operatorname{Hog} 1 \mathrm{P}_{2 \text { nuc }}$. mRNA is exported from the nucleus to the cytoplasm $\left(v_{\mathrm{ex}}\right)$, where it serves as template for protein synthesis and eventually will be degraded $\left(v_{\mathrm{rd}}\right)$. The dynamics of the enzyme concentrations is determined by translation $\left(v_{\mathrm{tl}}\right)$ and proteolysis $\left(v_{\mathrm{pd}}\right)$.

Glycerol production and carbohydrate metabolism (Supplementary Table 4 online). For the pathway from glucose to glycerol, expression of genes encoding the following enzymes is induced upon hyperosmotic shock: Glk1 and Hxk1 (glucokinase and hexokinase isoform I), as well as Gpd1, Gpp1 and Gpp2. Comprehensive models of yeast carbohydrate metabolism have been reported previously $y^{37,39-41}$. For the purpose of this study we combined several metabolic reactions taking into account gene expression changes: glucose uptake $\left(v_{1}\right)$, sugar phosphorylation $\left(v_{2}\right)$, phosphoglucoisomerase and phosphofructokinase $\left(v_{3}\right)$, aldolase $\left(v_{4}\right)$, triosephosphate isomerase $\left(v_{5}\right)$, the lower part of glycolysis from glyceraldehyde-3-phosphate to pyruvate $\left(v_{6}\right)$, the TCA cycle $\left(v_{7}\right)$, formation of ethanol from pyruvate $\left(v_{8}\right)$, two branches towards biosynthesis of biomass $\left(v_{9}, v_{10}\right)$ and NADH consumption and ATP formation in the respiratory chain $\left(v_{14}\right)$. Formation of glycerol and glycerol export are described by reactions $\left(v_{11}-v_{13}\right)$. We considered cellular consumption processes for $\mathrm{NAD}^{+}$ $\left(v_{15}\right)$ and ATP $\left(v_{16}\right)$. The kinetics were chosen to allow for stable steady-state concentrations and fluxes as previously determined ${ }^{37,41}$.

The osmo-regulated aquaglyceroporin Fps1 contributes to the control of intracellular glycerol level ${ }^{33,42}$. We assume that the channel is open when the turgor pressure assumes its original value and that transport is downregulated with fading $\Pi_{t}$, reaching zero when turgor pressure is completely lost.

$$
k_{13}(t)=k_{13}^{0} \cdot\left(\frac{\Pi_{t}(t)}{\Pi_{t}^{0}}\right)^{n_{2}}
$$

Equation (2) has been chosen in analogy with equation (1).

Biophysical changes (Supplementary Table 5 online). Under normal osmotic conditions the cell is characterized by its volume $V$ and by the internal and external osmotic pressure, $\Pi_{i}$ and $\Pi_{e}$, respectively. Since cells are filled with matter, a significant fraction of the volume given by $V_{b}=b \cdot V$ is not affected by osmotic changes. Starting from normal physiological conditions with given values of $V, \Pi_{i}, \Pi_{e}$ and turgor pressure $\Pi_{t}$, exposure to hyperosmotic stress (increase in $\Pi_{e}$ ) results in water outflow. In turn, this results in decreasing turgor pressure and volume. An increase of the intracellular osmolarity by glycerol accumulation drives water back into the cell to regain volume and turgor.

The mathematical description of the dynamics of volume and pressure is based on a detailed thermodynamic analysis of yeast cell osmoregulation $38,43,44$. According to the Boyle-Van't-Hoff relation the internal osmotic pressure is given by:

$$
\Pi_{i}=R T \frac{n}{V_{o s}}
$$

( $R$, gas constant; $T$, temperature; $n$, apparent number of osmotically active moles per cell; $\left.V_{o s}=V-V_{b}\right) . \Pi_{i}, V$ and $n$ are functions of time. Turgor pressure $\Pi_{t}$ is the hydrostatic pressure difference, which balances the osmotic pressure difference between cell interior and exterior. The cell actively maintains turgor pressure during osmoregulation. In the initial steady state (denoted by ' 0 ') we have

$$
\Pi_{t}^{0}=\Pi_{i}^{0}-\Pi_{e}^{0}
$$

The temporal evolution of $\Pi_{i}$ is calculated as the time derivative of equation (3):

$$
\frac{d}{d t} \Pi_{i}(t)=\Pi_{i}(t)\left(\frac{n^{\prime}(t)}{n(t)}-\frac{V_{o s}^{\prime}(t)}{V_{o s}(t)}\right) .
$$

The changes of $V_{o s}(t)$ and $\Pi_{i}(t)$ depend on the flow of water through the membrane. If only water is exchanged, the volume flow of water, $J_{w}(t)$, is proportional to the change of volume:

$$
J_{w}(t)=-\frac{d V_{o s}(t)}{d t}
$$

According to the principles of irreversible thermodynamics ${ }^{38,45}$ water flow can be related to the osmotic pressure difference as

$$
J_{w}(t)=G \cdot L_{p}\left(\Pi_{t}(t)+\Pi_{e}(t)-\Pi_{i}(t)\right),
$$

where $L_{p}$ is the hydraulic membrane permeability and $G=(4 \pi)^{1 / 3}(3 V)^{2 / 3}$ is a geometrical factor relating the volume of a sphere (the cells) to its surface.

The dependence of the turgor pressure on the actual volume was described by an empirical equation, $\Delta \Pi_{t}=\varepsilon \Delta V / V^{\Pi_{t}=0}$ (refs. 46,47) for isolated cells. The constant $\varepsilon$ is the volumetric elastic modulus. As an approximation we applied this formula to the yeast cell, although we note that there are examples where $\varepsilon$ is a function of volume or turgor pressure. Considering that turgor pressure collapses when the volume drops to $V^{\Pi_{t}=0}$, the temporal evolution of $\Pi_{t}$ reads

$$
\Pi_{t}(t)=\Pi_{t}^{0} \cdot\left(1-\frac{\left(V_{o s}^{0}-V_{o s}(t)\right)}{\left(V_{o s}^{0}-V^{\Pi_{t}=0}\right)}\right),
$$

for $V_{o s}(t)>V^{\Pi_{t}=0}$, otherwise $\Pi_{t}(t)=0$.

The passive response presents an initial decrease in both volume and turgor pressure potentially followed by additional volume decrease. Equation (8) implies a linear dependence of turgor pressure and cell volume. If the increase of $\Pi_{e}(t)$ is sufficiently strong that it leads to a complete loss in $\Pi_{t}(t)$, further efflux of water can be compensated only by volume decrease (smaller than $\left.V^{\Pi_{t}=0}\right)$. 
To account for the change of concentrations due to volume changes we considered the concentration $c$ of any substance as the number of moles $n$ per volume $V(c=n / V)$. In the calculation of osmotic pressure, change of $c$ is caused by the change of glycerol levels, since the cellular response is mainly due to a rise of the intracellular glycerol concentration ${ }^{48}$.

Connection of modules. The output signal of the phosphorelay system, Ssk1, was assigned as input signal for the MAP kinase module. Its output, $\mathrm{Hog} 1 \mathrm{P}_{2 \text { nuc }}$ served as input for the activation of gene expression. The production of mRNA and proteins (enzymes) led to a response of carbohydrate metabolism. Fps1 closed to allow for glycerol accumulation. The metabolic response of the cell was coupled to the osmotic response (volume and pressure changes) by considering glycerol as the osmotically active compound entering equation (5). All concentrations were linked via their relative changes to the variation of the cell volume, $V_{o s}$, which depends on osmotic alterations.

Note: Supplementary information is available on the Nature Biotechnology website.

\section{ACKNOWLEDGMENTS}

E.K. is supported by the Berlin Center of Genome Based Bioinformatics (BCB), financed by the German Federal Ministry for Education and Research (BMBF, grant 031U109C). B.N. and P.G. are PhD students of the National Research School for Genomics and Bioinformatics, Göteborg, supported by the Swedish Ministry for Education and Research. S.H. holds a research position of the Swedish Research Council. Research in his lab is supported by the European Commission (contracts QLK3-CT2000-00778 and QLK1-CT2001-01066) and 을 the Human Frontier Science Program. Systems Biology of yeast osmoregulation is supported by the European Commission (the QUASI project, contract LSHGCT2003-503230 to S.H. and E.K.).

\section{COMPETING INTERESTS STATEMENT}

The authors declare that they have no competing financial interests.

Received 14 February; accepted 10 May 2005

Published online at http://www.nature.com/naturebiotechnology/

1. Somero, G.N. \& Yancey, P.H. in Handbook of Physiology (eds. Hoffmann, J.F. \& Jamieson, J.D.) 441-484, (Oxford University Press, Oxford, New York, 1997).

2. Hohmann, S. Osmotic stress signaling and osmoadaptation in yeasts. Microbiol. Mol. Biol. Rev. 66, 300-372 (2002).

3. de Nadal, E., Alepuz, P.M. \& Posas, F. Dealing with osmostress through MAP kinase activation. EMBO Rep. 3, 735-740 (2002).

4. Reiser, V., Raitt, D.C. \& Saito, H. Yeast osmosensor $\operatorname{SIn} 1$ and plant cytokinin receptor Cre1 respond to changes in turgor pressure. J. Cell Biol. 161, 1035-1040 (2003).

5. Batchelor, E. \& Goulian, M. Robustness and the cycle of phosphorylation and dephosphorylation in a two-component regulatory system. Proc. Natl. Acad. Sci. USA 100, 691-696 (2003).

6. Tyson, J.J., Chen, K. \& Novak, B. Network dynamics and cell physiology. Nat. Rev. Mol. Cell Biol. 2, 908-916 (2001).

7. Schoeberl, B., Eichler-Jonsson, C., Gilles, E.D. \& Muller, G. Computational modeling of the dynamics of the MAP kinase cascade activated by surface and internalized EGF receptors. Nat. Biotechnol. 20, 370-375 (2002).

8. Kitano, H. Computational systems biology. Nature 420, 206-210 (2002).

9. Kofahl, B. \& Klipp, E. Modelling the dynamics of the yeast pheromone pathway. Yeast 21, 831-850 (2004).

10. Klipp, E., Herwig, R., Kowald, A., Wierling, C. \& Lehrach, H. Systems Biology in Practice Concepts, Implementation and Application (Wiley-VCH Verlag GmbH \& Co. KGaA, Weinheim, 2005).

11. Levchenko, A., Bruck, J. \& Sternberg, P.W. Scaffold proteins may biphasically affect the levels of mitogen-activated protein kinase signaling and reduce its threshold properties. Proc. Natl. Acad. Sci. USA 97, 5818-5823 (2000).

12. Ferrell, J.E., Jr. Self-perpetuating states in signal transduction: positive feedback double-negative feedback and bistability. Curr. Opin. Cell Biol. 14, 140-148 (2002).

13. Llorens, M., Nuno, J.C., Rodriguez, Y., Melendez-Hevia, E. \& Montero, F. Generalization of the theory of transition times in metabolic pathways: a geometrical approach. Biophys. J. 77, 23-36 (1999).

14. Heinrich, R., Neel, B.G. \& Rapoport, T.A. Mathematical models of protein kinase signal transduction. Mol. Cell 9, 957-970 (2002).

15. Huang, C.Y. \& Ferrell, J.E., Jr. Ultrasensitivity in the mitogen-activated protein kinase cascade. Proc. Natl. Acad. Sci. USA 93, 10078-10083 (1996).

16. Bhalla, U.S. \& lyengar, R. Emergent properties of networks of biological signaling pathways. Science 283, 381-387 (1999).

17. Kholodenko, B.N. Negative feedback and ultrasensitivity can bring about oscillations in the mitogen-activated protein kinase cascades. Eur. J. Biochem. 267, 1583-1588 (2000)
18. Asthagiri, A.R. \& Lauffenburger, D.A. A computational study of feedback effects on signal dynamics in a mitogen-activated protein kinase (MAPK) pathway model. Biotechnol. Prog. 17, 227-239 (2001).

19. Sedaghat, A.R., Sherman, A. \& Quon, M.J. A mathematical model of metabolic insulin signaling pathways. Am. J. Physiol. Endocrinol. Metab. 283, E1084-E1101 (2002).

20. Hartwell, L.H., Hopfield, J.J., Leibler, S. \& Murray, A.W. From molecular to modular cell biology. Nature 402, C47-C52 (1999).

21. O'Rourke, S.M. \& Herskowitz, I. Unique and redundant roles for HOG MAPK pathway components as revealed by whole-genome expression analysis. Mol. Biol. Cel/ 15, 532542 (2004).

22. Camisard, V., Brienne, J.P., Baussart, H., Hammann, J. \& Suhr, H. Inline characterization of cell concentration and cell volume in agitated bioreactors using in situ microscopy: application to volume variation induced by osmotic stress. Biotechnol. Bioeng. 78, 73-80 (2002).

23. Albertyn, J., Hohmann, S. \& Prior, B.A. Characterization of the osmotic-stress response in Saccharomyces cerevisiae: osmotic stress and glucose repression regulate glycerol3-phosphate dehydrogenase independently. Curr. Genet. 25, 12-18 (1994).

24. Saito, H. \& Tatebayashi, K. Regulation of the osmoregulatory HOG MAPK cascade in yeast. J. Biochem. 136, 267-272 (2004).

25. Rep, M., Albertyn, J., Thevelein, J.M., Prior, B.A. \& Hohmann, S. Different signalling pathways contribute to the control of GPD1 gene expression by osmotic stress in Saccharomyces cerevisiae. Microbiol. 145, 715-727 (1999).

26. Van Wuytswinkel, O. et al. Response of Saccharomyces cerevisiae to severe osmotic stress: evidence for a novel activation mechanism of the HOG MAP kinase pathway. Mol. Microbiol. 37, 382-397 (2000).

27. Ansell, R., Granath, K., Hohmann, S., Thevelein, J.M. \& Adler, L. The two isoenzymes for yeast NAD+-dependent glycerol 3-phosphate dehydrogenase encoded by GPD1 and GPD2 have distinct roles in osmoadaptation and redox regulation. EMBO J. 16, 2179 2187 (1997).

28. Krantz, M. et al. Anaerobicity prepares Saccharomyces cerevisiae cells for faster adaptation to osmotic shock. Eukaryot. Cell 3, 1381-1390 (2004).

29. Maeda, T., Wurgler-Murphy, S.M. \& Saito, H. A two-component system that regulates an osmosensing MAP kinase cascade in yeast. Nature 369, 242-245 (1994).

30. Hornberg, J.J. et al. Principles behind the multifarious control of signal transduction. ERK phosphorylation and kinase/phosphatase control. FEBS J. 272, 244-258 (2005).

31. Lu, J.M., Deschenes, R.J. \& Fassler, J.S. Saccharomyces cerevisiae histidine phosphotransferase Ypd1p shuttles between the nucleus and cytoplasm for SLN1-dependent phosphorylation of Ssk1p and Skn7p. Eukaryot. Cell 2, 1304-1314 (2003).

32. Hwang, I. \& Sheen, J. Two-component circuitry in Arabidopsis cytokinin signal transduction. Nature 413, 383-389 (2001).

33. Tamas, M.J. et al. Fps1p controls the accumulation and release of the compatible solute glycerol in yeast osmoregulation. Mol. Microbiol. 31, 1087-1104 (1999).

34. Pettersson, H., Filipsson, C., Becit, E., Brive, L. \& Hohmann, S. Aquaporins in yeasts and filamentous fungi. Biol. Cell. 97, 487-500 (2005).

35. Wurgler-Murphy, S.M., Maeda, T., Witten, E.A. \& Saito, H. Regulation of the Saccharomyces cerevisiae HOG1 mitogen-activated protein kinase by the PTP2 and PTP3 protein tyrosine phosphatases. Mol. Cell. Biol. 17, 1289-1297 (1997).

36. Karlgren, S. et al. Conditional osmotic stress in yeast: a system to study transport through aquaglyceroporins and osmostress signaling. J. Biol. Chem. 280, 7186-7193 (2005).

37. Rizzi, M., Baltes, M., Theobald, U. \& Reuss, M. In vivo analysis of metabolic dynamics in Saccharomyces cerevisiae: II. Mathematical model. Biotechnol. Bioeng. 55, 39-54 (1997).

38. Martinez de Maranon, I., Marechal, P.A. \& Gervais, P. Passive response of Saccharomyces cerevisiae to osmotic shifts: cell volume variations depending on the physiological state. Biochem. Biophys. Res. Commun. 227, 519-523 (1996).

39. Hynne, F., Dano, S. \& Sorensen, P.G. Full-scale model of glycolysis in Saccharomyces cerevisiae. Biophys. Chem. 94, 121-163 (2001).

40. Teusink, B. et al. Can yeast glycolysis be understood in terms of in vitro kinetics of the constituent enzymes? Testing biochemistry. Eur. J. Biochem. 267, 5313-5329 (2000).

41. Theobald, U., Mailinger, W., Baltes, M., Rizzi, M. \& Reuss, M. In vivo analysis of metabolic dynamics in Saccharomyces cerevisiae: I. Experimental observations. Biotechnol. Bioeng. 55, 305-316 (1997).

42. Tamas, M.J., Rep, M., Thevelein, J.M. \& Hohmann, S. Stimulation of the yeast high osmolarity glycerol (HOG) pathway: evidence for a signal generated by a change in turgor rather than by water stress. FEBS Lett. 472, 159-165 (2000).

43. Gervais, P. \& Beney, L. Osmotic mass transfer in the yeast Saccharomyces cerevisiae. Cell Mol. Biol. (Noisy-le-grand) 47, 831-839 (2001).

44. Gervais, P., Molin, P., Marechal, P.A. \& Herail-Foussereau, C. Thermodynamics of yeast cell osmoregulation: passive mechanisms. J. Biol. Phys. 22, 73-86 (1996).

45. Kedem, O. \& Katchalsky, A. Thermodynamic analysis of the permeability of biological membranes to non-electrolytes. Biochim. Biophys. Acta 27, 229-246 (1958).

46. Caley, S.D., Guttman, H.J. \& Record, M.T.J. Biophysical characterization of changes in amounts and activity of Escherichia coli cell and compartment water and turgor pressure in response to osmotic stress. Biophys. J. 78, 1748-1764 (2000).

47. Zimmermann, U. Physics of turgor- and osmoregulation. Ann. Rev. Plant Physiol. 29, 121-148 (1978).

48. Reed, R.H., Chudek, J.A., Foster, R. \& Gadd, G.M. Osmotic significance of glycerol accumulation in exponentially growing yeasts. Appl. Environ. Microbiol. 53, 2119-2123 (1987). 